\title{
Double Schubert polynomials for the classical Lie groups
}

\author{
Takeshi Ikeda $^{1}$ and Leonardo Mihalcea ${ }^{2}$ and Hiroshi Naruse ${ }^{3}$ \\ ${ }^{1}$ Okayama University of Science, Japan \\ ${ }^{2}$ Duke University, U.S.A. \\ ${ }^{3}$ Okayama University, Japan
}

\begin{abstract}
For each infinite series of the classical Lie groups of type $B, C$ or $D$, we introduce a family of polynomials parametrized by the elements of the corresponding Weyl group of infinite rank. These polynomials represent the Schubert classes in the equivariant cohomology of the corresponding flag variety. They satisfy a stability property, and are a natural extension of the (single) Schubert polynomials of Billey and Haiman, which represent non-equivariant Schubert classes. When indexed by maximal Grassmannian elements of the Weyl group, these polynomials are equal to the factorial analogues of Schur $Q$ - or $P$-functions defined earlier by Ivanov.

Résumé Pour chaque serie infinie des groupe de Lie classiques de type $B, C$ ou $D$, nous preséntons une famille de polynômes indexées par de élèments de groupe de Weyl correspondant de rang infini. Ces polynômes représent des classes de Schubert dans la cohomologie équivariante des variétés de drapeaux. Ils ont une certain proprieté de stabilité, et ils extendent naturellement des polynômes Schubert (simples) de Billey et Haiman, que represent des classes de Schubert dans la cohomologie non-équivariante. Quand ils sont indexées par des élèments Grassmanniennes de groupes de Weyl, ces polynômes sont equales avec des analogues factorielles de fonctions Q et P de Schur, que été studié en avant d'Ivanov.
\end{abstract}

Keywords: Schubert polynomial, flag variety, factorial Q-function

\section{Introduction}

This article is an extended abstract of (IMN). Details of proofs and some results are omitted.

We are concerned with the problem of finding natural polynomials that represent the cohomology classes of Schubert varieties in generalized flag varieties. This problem goes back at least to the 1970s, when Bernstein, Gelfand, and Gelfand (BGG) and Demazure (D) showed that, for general $G / B$ 's, one can construct polynomial representatives of the Schubert classes by using the so-called divided difference operators $\partial_{i}$. For the general linear group, Lascoux and Schützenberger (LS) discovered a particularly important family of polynomials defined by applying the divided difference operators to a special monomial of top degree, which represents the point class. These polynomials are called Schubert polynomials and have many nice properties.

It is a natural problem to extend this theory to the other classical Lie types. Fomin and Kirillov (FK) listed up five important properties that characterize the Schubert polynomials. Moreover they showed it is 1365-8050 (C) 2008 Discrete Mathematics and Theoretical Computer Science (DMTCS), Nancy, France 
impossible to construct a theory of 'Schubert polynomials' in types $B_{n}, C_{n}$ and $D_{n}$ satisfying the same properties. For type $B_{n}$, they constructed several families of polynomials which satisfy all but one of these properties. On the other hand, for each classical Lie types, Billey and Haiman (BH) constructed a family of 'polynomials' which are identified with elements in an inverse system of cohomology ring of flag variety when $n$ goes to infinity. Here we should be careful that the meaning of 'polynomial' is a bit different from those in (FK) (for the precise meaning, see below). These polynomials satisfy an infinite system of divided difference equations and are stable under inclusion into an infinite union of Weyl groups. In (FK) they notice that the limit of a stable version of their polynomials coincide with the ones in (BH).

Our goal is to construct and study a double version of Billey and Haiman's polynomials. Type $A$ double Schubert polynomials of Lascoux and Schützenberger (LS), (L) admit at least two (related) geometric interpretations. Firstly, a theorem of Fulton (F1) states that a degeneracy loci of morphisms between vector bundles coincides with the double Schubert polynomials. Secondly one can identify the double Schubert polynomials with the Schubert classes in equivariant cohomology ring of the flag variety of type $A$. Such geometric interpretations for the double Schubert polynomials were obtained in the context of Thom polynomials by Fehér and Rimányi (FR). In (B2) (§8) Billey noticed that specializing double Schubert polynomials yields the equivariant localization properties (cf. (2)).

The first point of view, degeneracy loci formulas, has been used by Fulton (F2) and also by Kresch and Tamvakis (KT) to construct candidates for double Schubert polynomials in types $B_{n}, C_{n}$ and $D_{n}$. The main difficulty in these approaches lie in the choice of "top" polynomials, the representative for the diagonal class of the flag bundle, that produce arbitrary double Schubert polynomials when we apply divided difference operators successively.

In this paper, we adopt the equivariant cohomology approach to construct three families of double Schubert polynomials in types $B, C$ and $D$. In particular, we will make full use of localization technique in equivariant cohomology theory, which do not exist in the non-equivariant case. The obtained polynomials have desired properties that inherits from the single (non-equivariant) Schubert polynomials of Billey and Haiman. One of the main feature of our polynomials is the stability property mentioned above. This is not the case for the previously known candidates in (F2) and (KT).

Now let us review the results of Billey and Haiman in more detail. For the sake of simplicity, we mainly consider type $C$ case. The main ingredient of their constructions is the ring of Schur $Q$-functions. For a good reference on the theory of Schur $Q$-functions consult (M). Let $Q_{\lambda}(x)$ be Schur's $Q$-functions corresponding to a strict partition $\lambda$. They form a distinguished $\mathbb{Z}$-basis of the ring $\Gamma$, a subring of the ring of 'symmetric functions'. These functions first appeared in the context of Schubert calculus when Pragacz (P) identified $Q_{\lambda}(x)$ 's with the Schubert classes for the Lagrangian Grassmannians. Billey and Haiman's polynomials live in the following extended ring of $\Gamma$ :

$$
R_{\infty}^{B H}:=\mathbb{Z}\left[z_{1}, z_{2}, \ldots\right] \otimes_{\mathbb{Z}} \Gamma .
$$

They identified $R_{\infty}^{B H}$ with a subring of the inverse limit of $H^{*}\left(\mathcal{F}_{n}\right)$ where $\mathcal{F}_{n}$ is the flag variety of order $n$ in type $C_{n}$. The Billey-Haiman polynomials $\mathfrak{C}_{w}(z ; x)$ are indexed by the elements $w$ in the infinite Weyl group $W_{\infty}=\bigcup_{n \geq 1} W_{n}$, where $W_{n}$ is the Weyl group of type $C_{n}$. By the construction $\mathfrak{C}_{w}(z ; x)$ has the stability properties. More precisely $\mathfrak{C}_{w}(z ; x)$ is identified with the inverse limit of Schubert classes corresponding to $w \in W_{\infty}$. Another important feature of these polynomials is that if $w$ is a maximal Grassmannian element in $W_{\infty}$ that corresponds to a strict partition $\lambda$, then $\mathfrak{C}_{w}(z ; x)$ coincides with $Q_{\lambda}(x)$ (there are analogous results for types $B, D$ ). This recovers the theorem of Pragacz. 
The first result of this paper is the existence and uniqueness of our polynomials (Theorem 3.1). Consider the ring $R_{\infty}=\mathbb{Z}\left[t_{1}, t_{2}, \ldots\right] \otimes_{\mathbb{Z}} R_{\infty}^{B H}$. We construct two commuting actions of $W_{\infty}$ on $R_{\infty}$ which give rise to two commuting famifies of divided difference operators $\left\{\partial_{i}\right\}_{i},\left\{\delta_{i}\right\}_{i}$ where $i=0,1,2, \ldots$ (see $\S 3.1$ for the definition). Here we just remark that $\partial_{0}, \delta_{0}$ act on $\Gamma$ in a nontrivial way. Our polynomials $\left\{\mathfrak{C}_{w}(z, t ; x)\right\}_{w}, w \in W_{\infty}$ are defined to be the unique solution of the following equations:

$$
\partial_{i} \mathfrak{C}_{w}=\left\{\begin{array}{ll}
\mathfrak{C}_{w s_{i}} & \text { if } \ell\left(w s_{i}\right)=\ell(w)-1 \\
0 & \text { if } \ell\left(w s_{i}\right)=\ell(w)+1
\end{array}, \quad \delta_{i} \mathfrak{C}_{w}=\left\{\begin{array}{ll}
\mathfrak{C}_{s_{i} w} & \text { if } \ell\left(s_{i} w\right)=\ell(w)-1 \\
0 & \text { if } \ell\left(s_{i} w\right)=\ell(w)+1
\end{array},\right.\right.
$$

combined with a normalization property, where $s_{i}$ are the simple reflections. By the construction, it is clear that $\mathfrak{C}_{w}(z, t ; x)$ specialize (when $t=0$ ) to Billey and Haiman's polynomials $\mathfrak{C}_{w}(z ; x)$. Moreover, for maximal Grassmannian elements of Weyl group, $\mathfrak{C}_{w}(z, t ; x)$ agree with the factorial $Q$-functions introduced by Ivanov (Iv). This result is a natural $T$-equivariant analogue of the theorem of Pragacz. Note that the fact has been established in (I) and (IN) by a different method.

The main technical feature of this paper is the systematic use of localization maps. Let $\mathcal{F}_{n}^{T}$ denotes the set of $T$-fixed points, which is naturally indexed by Weyl group $W_{n}$. By the pullback morphism induced by the inclusion $\mathcal{F}_{n}^{T} \hookrightarrow \mathcal{F}_{n}$, the equivariant cohomology $H_{T}^{*}\left(\mathcal{F}_{n}\right)$ is considered to be a subring of $H_{T}^{*}\left(\mathcal{F}_{n}^{T}\right)=\prod_{v \in W_{n}} H_{T}^{*}\left(e_{v}\right)=\prod_{v \in W_{n}} \mathbb{Z}\left[t_{1}, \ldots, t_{n}\right]$, where $e_{v}$ is the $T$-fixed point corresponding to $v \in W_{n}$. Therefore a class $\eta$ in $H_{T}^{*}\left(\mathcal{F}_{n}\right)$ can be determined by giving their restriction $\left.\eta\right|_{e}$ to any $T$-fixed point $e_{v}, v \in W_{n}$. To describe the restriction we introduce ring homomorphims ( $\$ 4.2$ )

$$
\Phi_{v}: R_{\infty} \longrightarrow \mathbb{Z}\left[t_{1}, t_{2}, \ldots\right]
$$

associated with $v$ in $W_{\infty}$. The equivariant Schubert class $\sigma_{w}^{T}$ (see $\S 4.1$ ) is calculated from our polynomial as

$$
\Phi_{v}\left(\mathfrak{C}_{w}(z, t ; x)\right)=\left.\sigma_{w}^{T}\right|_{v},
$$

where $\sigma_{w}^{T}$ in the right hand side can be thought as an element in $H_{T}^{*}\left(\mathcal{F}_{n}\right)$ for all $n$ such that $w, v \in W_{n}$. In this sense, our polynomials represent the equivariant Schubert classes. Note that as Arabia (A) showed $\left.\sigma_{w}^{T}\right|_{e_{v}}$ are nothing but $\xi$-functions of Kostant and Kumar (KK).

Our method to prove the existence of the double Schubert polynomials relies on the Goresky-KottwitzMacPherson description of the equivariant cohomology (GKM). The main idea is to consider a ring $H_{\infty}$ as a subring of $\prod_{v \in W_{\infty}} \mathbb{Z}\left[t_{1}, t_{2}, \ldots\right]$ which consists of elements satisfying the GKM conditions. We have a natural $\mathbb{Z}\left[t_{1}, t_{2}, \ldots\right]$-basis $\sigma_{w}^{(\infty)}$ indexed by the elements of $W_{\infty}$. These element behave in the similar way as $\mathfrak{C}_{w}(z, t ; x)$ do under the naturally defined operators $\partial_{i}, \delta_{i}$ on $H_{\infty}$ (cf. (1)). We will show that the localization map $\Phi=\left(\Phi_{v}\right)_{v \in W_{\infty}}$ gives the isomorphism of rings from $R_{\infty}$ onto $H_{\infty}$. The injectivity and the fact that $\operatorname{Im}\left(R_{\infty}\right) \subset H_{\infty}$ are rather easy to prove. To prove the surjectivity we first show that the image of $Q_{\lambda}(x \mid t)$ under $\Phi$ is $\sigma_{w_{\lambda}}^{(\infty)}$, where $w_{\lambda}$ is the maximal Grassmannian element corresponding to $\lambda$. This can be shown by using a vanishing property that characterizes the factorial Schur functions and also the equivariant Schubert classes. Then we solve a recursive formula, the equivariant version of transition equations of Billey (B1), to get $\Phi^{-1}\left(\sigma_{w}^{(\infty)}\right)$ in $R_{\infty}$ for arbitrary $w \in W_{\infty}$. Set $\mathfrak{C}_{w}(z, t ; x)=\Phi^{-1}\left(\sigma_{w}^{(\infty)}\right)$. Then the defining equation for $\mathfrak{C}_{w}(z, t ; x)$ is satisfied thanks to a commutativity property of the divided differences and $\Phi$. 
The main combinatorial upshot is the following explicit formula. Let $w_{0}^{(n)}$ be the longest element in the Weyl group $W_{n}$ of type $C_{n}$. Then the double Schubert polynomial corresponding to $w_{0}^{(n)}$ is given by

$$
\mathfrak{C}_{w_{0}^{(n)}}(z, t ; x)=Q_{2 n-1,2 n-3, \ldots, 3,1}\left(x \mid-z_{1}, t_{1},-z_{2}, t_{2}, \ldots,-z_{n-1}, t_{n-1}\right) .
$$

For type $D$ case, we have

$$
\mathfrak{D}_{w_{0}^{(n)}}(z, t ; x)=P_{2 n-2,2 n-4, \ldots, 4,2}\left(x \mid-z_{1}, t_{1},-z_{2}, t_{2}, \ldots,-z_{n-1}, t_{n-1}\right),
$$

where $w_{0}^{(n)}$ denotes the longest element in $W\left(D_{n}\right)$ and we use the same convention of the factorial $P$ function $P_{\lambda}(x \mid t)$ as in (IN). We prove these formulas using the behavior of $Q_{\lambda}(x \mid t)$ or $P_{\lambda}(x \mid t)$ under the action of $\delta_{i}$. In fact if we denote the function in right hand side of (3) by $\Delta_{n}=\Delta_{n}(z, t ; x)$, then we can show

$$
\delta_{n-1} \cdots \delta_{1} \delta_{0} \delta_{1} \cdots \delta_{n-1} \Delta_{n}=\Delta_{n-1} .
$$

Moreover we can directly verify $\Phi_{v}\left(\Delta_{n}\right)=\left.\sigma_{w_{0}^{(n)}}^{T}\right|_{e}$ for $v \in W_{n}$. Combined with these two facts we can prove that if $w \in W_{n}$ then $F_{w}=\partial_{w^{-1}} w_{0}^{(n)} \Delta_{n}$ does not depend on $n$ and $\left\{F_{w}\right\}_{w \in W_{\infty}}$ satisfies the divided difference equations. Then by the uniqueness of solution we have $F_{w}=\mathfrak{C}_{w}(z, t ; x)$ for all $w \in W_{\infty}$. In particular we have $\mathfrak{C}_{w_{0}^{(n)}}=\Delta_{n}$.

One motivation of the present paper was to give a geometric interpretation to the factorial Schur $Q$ function by means of degeneracy loci formulas. For type $A$ case this problem was treated in (Mi), where Kempf-Laksov formula for degeneracy loci is identified with the Jacobi-Trudi type formula for the factorial (ordinary) Schur function. We also have a formula that express the factorial $Q$-function as a multi Schur Pfaffian. This comes from a Lagrangian degeneracy loci formula of Kazarian (Ka) as an analogy of Kempf-Laksov formula. This matter will be discussed in the full paper.

While our results generalize to all classical Lie types, we will restrict ourselves mainly to type $C$ for the remainder of this article.

\section{Preliminaries}

\subsection{Infinite hyperoctahedral groups}

The infinite hyperoctahedral group $W_{\infty}$ consists of all permutations $w$ of the set $\{1,2, \ldots\} \cup\{\overline{1}, \overline{2}, \ldots\}$ such that $w(i) \neq i$ for only finitely many $i$, and $\overline{w(i)}=w(\bar{i})$ for all $i$. Each $w \in W_{\infty}$ is considered to be a signed (barred) permutation of $\{1,2, \ldots\}$. We often use one-line notation $w=(w(1), w(2), \ldots)$ to denote an element $w \in W_{\infty}$. The transpositions

$$
s_{0}=(1, \overline{1}), \quad s_{i}=(i+1, i) \quad(i>0)
$$

are standard generators satisfying the relations:

$$
s_{i}^{2}=e \quad \text { for all } \quad i, \quad s_{0} s_{1} s_{0} s_{1}=s_{1} s_{0} s_{1} s_{0}, \quad s_{i} s_{i+1} s_{i}=s_{i+1} s_{i} s_{i+1} \quad \text { for } \quad i>0 .
$$

By this set of generators we can think of $\left(W_{\infty}, S\right)$ as a Coxeter system, where $S=\{0,1,2, \ldots\}$. In particular we have the Bruhat-Chevalley order on $W_{\infty}$. The subgroup $W_{n}=\left\langle s_{0}, s_{1}, \ldots, s_{n-1}\right\rangle$ is isomorphic to the Weyl group of type $C_{n}$. We have $W_{\infty}=\bigcup_{n \geq 1} W_{n}$. The Bruhat-Chevalley order of $W_{n}$ makes it a sub-poset of $W_{\infty}$. 


\subsection{The ring of $Q$-functions}

Our main reference for symmetric function is (M). Let $\Lambda$ denote the ring of symmetric functions in infinitely many indeterminates $x=\left(x_{1}, x_{2}, \ldots\right)$. The Schur's $Q$-function $Q_{\lambda}(x)$ is an element of $\Lambda$ indexed by a strict partition $\lambda=\left(\lambda_{1}>\cdots>\lambda_{r}>0\right)$. Define $Q_{i}(x)$ as the coefficient of $u^{i}$ in the generating function

$$
f(u)=\prod_{i=1}^{\infty} \frac{1+x_{i} u}{1-x_{i} u}=\sum_{k \geq 0} Q_{k}(x) u^{k} .
$$

Note that $Q_{0}=1$. The identity $f(u) f(-u)=1$ yields

$$
Q_{i}(x)^{2}+2 \sum_{j=1}^{i}(-1)^{j} Q_{i+j}(x) Q_{i-j}(x)=0 \quad \text { for } \quad i>0 .
$$

It is known that the ideal of relations among the functions $Q_{k}(x)$ is generated by the previous relations. For $i \geq j \geq 0$, define elements

$$
Q_{i, j}(x):=Q_{i}(x) Q_{j}(x)+2 \sum_{k=1}^{j}(-1)^{k} Q_{i+k}(x) Q_{j-k}(x) .
$$

Note that $Q_{i, 0}(x)=Q_{i}(x)$ and $Q_{i, i}(x)(i>0)$ is identically zero. Let $\mathcal{S P}$ denote the set of all strict partitions. For $\lambda$ in $\mathcal{S P}$ we write $\lambda=\left(\lambda_{1}>\lambda_{2}>\cdots>\lambda_{r} \geq 0\right)$ with $r$ even. Then the corresponding Schur's $Q$-function $Q_{\lambda}=Q_{\lambda}(x)$ is defined by

$$
Q_{\lambda}(x)=\operatorname{Pf}\left(Q_{\lambda_{i}, \lambda_{j}}(x)\right)_{1 \leq i<j \leq r},
$$

where Pf denote the Pfaffian.

Set $\Gamma$ to be the subring of $\Lambda$ defined by $\Gamma:=\mathbb{Z}\left[Q_{1}(x), Q_{2}(x), \ldots\right]$. It is known then that the functions $Q_{\lambda}(x)$ for $\lambda \in \mathcal{S P}$ form a $\mathbb{Z}$-basis of $\Gamma$.

\section{Double Schubert polynomials of type $C$}

\subsection{Divided difference operators}

Set

$$
R_{\infty}:=\mathbb{Z}\left[t_{1}, t_{2}, \ldots\right] \otimes_{\mathbb{Z}} \mathbb{Z}\left[z_{1}, z_{2}, \ldots\right] \otimes_{\mathbb{Z}} \Gamma
$$

We make $W_{\infty}$ act on $R_{\infty}$ by letting $s_{i}^{z}$ interchange $z_{i}$ and $z_{i+1}$, for $i>0$, and letting $s_{0}^{z}$ replace $z_{1}$ and $-z_{1}$, and also

$$
s_{0}^{z} Q_{k}(x)=Q_{k}(x)+2 \sum_{j=1}^{k} z_{1}^{j} Q_{k-j}(x) .
$$

We use another action of $W_{\infty}$ given by letting $s_{i}^{t}$ interchange $t_{i}$ and $t_{i+1}$, for $i>0$, and letting $s_{0}^{t}$ replace $t_{1}$ and $-t_{1}$, and also

$$
s_{0}^{t} Q_{k}(x)=Q_{k}(x)+2 \sum_{j=1}^{k}\left(-t_{1}\right)^{j} Q_{k-j}(x) .
$$


We define divided difference operators on $R_{\infty}$

$$
\partial_{0} f=\frac{f-s_{0}^{z} f}{-2 z_{1}}, \quad \partial_{i} f=\frac{f-s_{i}^{z} f}{-\left(z_{i+1}-z_{i}\right)}, \quad \delta_{0} f=\frac{f-s_{0}^{t} f}{2 t_{1}} \quad \delta_{i} f=\frac{f-s_{i}^{t} f}{t_{i+1}-t_{i}} \quad \text { for } \quad i>0 .
$$

Define also

$$
\partial_{\hat{1}} f=\frac{f-s_{\hat{1}}^{z} f}{-\left(z_{2}+z_{1}\right)}, \quad \delta_{\hat{1}} f=\frac{f-s_{\hat{1}}^{t} f}{t_{2}+t_{1}},
$$

where $s_{\hat{1}}^{z}=s_{0}^{z} s_{1}^{z} s_{0}^{z}$ and $s_{\hat{1}}^{t}=s_{0}^{t} s_{1}^{t} s_{0}^{t}$.

Remark. We introduced $x=\left(x_{1}, x_{2}, \ldots\right)$ as algebraically independent variables from $z=\left(z_{1}, z_{2}, \ldots\right)$ and $t=\left(t_{1}, t_{2}, \ldots\right)$. However it is natural to impose the following relation ( $\left.\mathrm{cf}(\mathrm{BH})\right)$ :

$$
p_{i}(x)=\frac{1}{2} p_{i}(t)-\frac{1}{2} p_{i}(z) \quad \text { for odd } \quad i>0,
$$

where $p_{i}$ denote the powersum symmetric function. Recall that the ring $\mathbb{Q} \otimes_{\mathbb{Z}} \Gamma$ is generated by algebraically independent elements $p_{1}(x), p_{3}(x), \ldots$ Our formulas of the action of $s_{0}^{z}$ and $s_{0}^{t}$ above are consistent with these relations.

\subsection{Definition and first properties}

We state the existence and uniqueness for the double Schubert polynomials of type $C$.

Theorem 3.1 There exist a unique family of elements $\left\{\mathfrak{C}_{w}\right\}_{w} \subset R_{\infty}, w \in W_{\infty}$, satisfying the equations

$$
\partial_{i} \mathfrak{C}_{w}=\left\{\begin{array}{l}
\mathfrak{C}_{w s_{i}} \text { if } \ell\left(w s_{i}\right)<\ell(w) \\
0 \quad \text { otherwise }
\end{array} \quad, \quad \delta_{i} \mathfrak{C}_{w}=\left\{\begin{array}{l}
\mathfrak{C}_{s_{i} w} \text { if } \ell\left(s_{i} w\right)<\ell(w) \\
0 \quad \text { otherwise }
\end{array},\right.\right.
$$

for all $i \geq 0$, together with the condition that the constant term of $\mathfrak{C}_{w}$ is zero except for $w=e$, and that $\mathfrak{C}_{e}=1$.

Example. The double Schubert polynomials $\mathfrak{C}_{w}$ for $w \in W_{2}$ are

$$
\begin{gathered}
\mathfrak{C}_{e}=1, \quad \mathfrak{C}_{s_{0}}=Q_{1}(x), \quad \mathfrak{C}_{s_{1}}=Q_{1}(x)+z_{1}-t_{1}, \\
\mathfrak{C}_{s_{1} s_{0}}=Q_{2}(x)-t_{1} Q_{1}(x), \quad \mathfrak{C}_{s_{0} s_{1}}=Q_{2}(x)+z_{1} Q_{1}(x), \\
\mathfrak{C}_{s_{0} s_{1} s_{0}}=Q_{2,1}(x), \quad \mathfrak{C}_{s_{1} s_{0} s_{1}}=Q_{3}(x)+\left(z_{1}-t_{1}\right) Q_{2}(x)-t_{1} z_{1} Q_{1}(x), \\
\mathfrak{C}_{s_{0} s_{1} s_{0} s_{1}}=Q_{3,1}(x)+\left(z_{1}-t_{1}\right) Q_{2,1}(x) .
\end{gathered}
$$

We collect here some basic properties of $\mathfrak{C}_{w}=\mathfrak{C}_{w}(z, t ; x)$.

Theorem 3.2 1. The double Schubert polynomials $\left\{\mathfrak{C}_{w}\right\}_{w}$ form a $\mathbb{Z}[t]$-basis of $R_{\infty}$.

2. For all $w \in W_{\infty}$ we have

$$
\mathfrak{C}_{w}(z, 0 ; x)=\mathfrak{C}_{w}(z ; x),
$$

where $\mathfrak{C}_{w}(z ; x)$ denotes Billey-Haiman's polynomial. 
3. (Symmetry) We have $\mathfrak{C}_{w}(-t,-z ; x)=\mathfrak{C}_{w^{-1}}(z, t ; x)$.

4. (Positivity) When we write

$$
\mathfrak{C}_{w}(z, t ; x)=\sum_{\lambda \in \mathcal{S} \mathcal{P}} f_{w, \lambda}(z, t) Q_{\lambda}(x)
$$

we have $f_{w, \lambda}(z, t) \in \mathbb{N}\left[-t_{1}, \ldots,-t_{n-1}, z_{1}, \ldots, z_{n-1}\right]$ if $w \in W_{n}$.

Set $P_{k}(x)=\frac{1}{2} Q_{k}(x)(k \geq 1)$. Define $\Gamma^{\prime}=\mathbb{Z}\left[P_{1}(x), P_{2}(x), \ldots\right]$ and $R_{\infty}^{\prime}=\mathbb{Z}\left[t_{1}, t_{2}, \ldots\right] \otimes_{\mathbb{Z}} \Gamma \otimes_{\mathbb{Z}}$ $\mathbb{Z}\left[z_{1}, z_{2}, \ldots\right]$. Let $W_{\infty}^{\prime}=\left\langle s_{\hat{1}}, s_{1}, s_{2}, \ldots\right\rangle$ be the infinite Weyl group of type $D$, where $s_{\hat{1}}=s_{0} s_{1} s_{0}$.

Theorem 3.3 There exist a unique family of elements $\left\{\mathfrak{D}_{w}\right\}_{w} \subset R_{\infty}^{\prime}, w \in W_{\infty}^{\prime}$, satisfying the equations

$$
\partial_{i} \mathfrak{D}_{w}=\left\{\begin{array}{l}
\mathfrak{D}_{w s_{i}} \text { if } \ell\left(w s_{i}\right)<\ell(w) \\
0 \quad \text { otherwise }
\end{array} \quad, \quad \delta_{i} \mathfrak{D}_{w}=\left\{\begin{array}{l}
\mathfrak{D}_{s_{i} w} \text { if } \ell\left(s_{i} w\right)<\ell(w) \\
0 \quad \text { otherwise }
\end{array},\right.\right.
$$

for all $i \geq 1$ and $i=\hat{1}$, together with the condition that the constant term of $\mathfrak{D}_{w}$ is zero except for $w=e$, and that $\mathfrak{D}_{e}=1$.

Type $B$ polynomials $\mathfrak{B}_{w}\left(w \in W_{n}\right)$ are defined to be $2^{-s(w)} \mathfrak{C}_{w}$, where $s(w)$ denotes the number of sign changes in $w$. There are also elements in $R_{\infty}^{\prime}$.

\subsection{Maximal Grassmannian elements}

An element $w \in W_{\infty}$ is a maximal Grassmannian element if $w(1)<w(2)<\cdots<w(i)<\cdots$ in the order $\cdots<\overline{3}<\overline{2}<\overline{1}<1<2<3<\cdots$. Let $W_{\infty}^{\max }$ denote the set of all maximal Grassmannian elements in $W_{\infty}$. For $w \in W_{\infty}^{\max }$ let $r$ be such that

$$
w(1)<\cdots<w(r)<1 \quad \text { and } \quad \overline{1}<w(r+1)<w(r+2)<\cdots .
$$

Set

$$
\lambda_{i}=\overline{w(i)} \text { for } \quad 1 \leq i \leq r .
$$

Then $\lambda=\left(\lambda_{1}, \ldots, \lambda_{r}\right)$ is a strict partition i.e., $\lambda_{1}>\cdots>\lambda_{r}>0$. Let $\mathcal{S P}$ denote the set of all strict partitions. The correspondence gives a bijection

$$
W_{\infty}^{\max } \longrightarrow \mathcal{S P} .
$$

Note that this bijection preserves the partial order when $\mathcal{S P}$ is considered to be a partially ordered set given by the inclusion $\lambda \subset \mu$ of strict partitions. Moreover if $v \in W_{\infty}^{\max }$ corresponds to $\lambda \in \mathcal{S P}$ we have $\ell(w)=|\lambda|$.

Theorem 3.4 If $w \in W_{\infty}$ is a maximal Grassmannian element corresponding to $\lambda$ in $\mathcal{S P}$ then

$$
\mathfrak{C}_{w}(z, t ; x)=Q_{\lambda}(x \mid t),
$$

where $Q_{\lambda}(x \mid t)$ is the factorial $Q$-function defined by Ivanov.

Remark. We have analogous result for types $B, D$ in terms of factorial $P$-functions (cf. (IN)).

Remark. The fact that factorial $Q$-function represents the equivariant Schubert class of the Lagrangian Grassmannian $L G_{n}$ was established in (I). Note that the subring $\mathbb{Z}[t] \otimes_{\mathbb{Z}} \Gamma$ of $R_{\infty}$ has a $\mathbb{Z}[t]$-basis consisting of $Q_{\lambda}(x \mid t), \lambda \in \mathcal{S P}$. 


\subsection{Longest elements of $W_{n}$}

Let $\lambda, \mu$ be partitions of length less than or equal to $r$. For sequences $c^{(i)}=\left(c_{0}^{(i)}, c_{1}^{(i)}, \ldots\right)(1 \leq i \leq r)$ of commutative elements, the multi-Schur determinant is defined by

$$
s_{\lambda / \mu}\left(c^{(1)}, \ldots, c^{(r)}\right)=\operatorname{det}\left(c_{\lambda_{i}-\mu_{j}+j-i}^{(i)}\right)_{1 \leq i, j \leq r}
$$

where $c_{k}^{(i)}=0$ for $k<0$. Let $h_{k}$ denote the complete symmetric function of degree $k$. Set $\rho_{k}=$ $(k, k-1, \ldots, 1)$.

Theorem 3.5 Let $w_{0}^{(n)}$ denote the longest element in $W_{n}$. We have

$$
\mathfrak{C}_{w_{0}^{(n)}}(z, t ; x)=\sum_{\lambda \subset \rho_{n-1}} Q_{\rho_{n}+\lambda}(x) s_{\rho_{n-1} / \lambda^{\prime}}\left(c^{(1)}, \ldots, c^{(n-1)}\right),
$$

where $c_{k}^{(i)}=h_{k}\left(z_{1}, \ldots, z_{i},-t_{1}, \ldots,-t_{i}\right)$ and $\lambda^{\prime}$ is the conjugate partition of $\lambda$.

We have an alternative expression for $\mathfrak{C}_{w_{0}^{(n)}}(z, t ; x)$ in terms of a factorial $Q$-function, i.e.

$$
\mathfrak{C}_{w_{0}^{(n)}}(z, t ; x)=Q_{\rho_{n}+\rho_{n-1}}\left(x \mid-z_{1}, t_{1},-z_{2}, t_{2}, \ldots,-z_{n-1}, t_{n-1}\right) .
$$

\section{Geometric interpretation}

In this section we explain the geometric meaning of our polynomials.

\subsection{Flag variety of type $C$}

Let $G_{n}$ denote the (complex) symplectic group of rank $n$ and $T_{n} \subset B_{n}$ be a maximal torus and a Borel subgroup. The flag variety is defined by $\mathcal{F}_{n}=G_{n} / B_{n}$. The Weyl group of $\left(G_{n}, T_{n}\right)$ is identified with $W_{n}$. For each $w \in W_{n}$, let $e_{w} \in \mathcal{F}_{n}$ denote the corresponding $T_{n}$-fixed point. Let $B_{n}^{-}$denote the opposite Borel subgroup of $B_{n}$. Define $\Omega_{w}=B_{n}^{-} e_{w}$. The Schubert variety is defined to be the closure $\bar{\Omega}_{w}$. Since $\bar{\Omega}_{w}$ is a $T_{n}$-stable closed subvariety we have the class $\sigma_{w}^{T_{n}}=\left[\bar{\Omega}_{w}\right]_{T_{n}} \in H_{T_{n}}^{2 \ell(w)}\left(\mathcal{F}_{n}\right)$, the equivariant Schubert class, where $\ell(w)$ is the length of $w \in W$.

\subsection{Localization maps}

Let $v \in W_{\infty}$. We make $W_{\infty}$ act on $\mathbb{Z}\left[t_{1}, t_{2}, \ldots\right]$ as signed permutations of the variables $t_{1}, t_{2}, \ldots$ We define a ring homomorphism $\Phi_{v}$ from $R_{\infty}$ to $\mathbb{Z}\left[t_{1}, t_{2}, \ldots\right]$ by

$$
\Phi_{v}(f(z, t ; x))=f\left(v(t), t ; t_{v}\right)
$$

where $v(t)=\left(v\left(t_{1}\right), v\left(t_{2}\right), \ldots\right)$ and $t_{v}$ is defined by

$$
\left(t_{v}\right)_{i}=\left\{\begin{array}{ll}
t \overline{v(i)} & \text { if } v(i)<1 \\
0 & \text { otherwise }
\end{array} .\right.
$$

The ring $H_{T_{n}}^{*}(p t)$ is identified with $\mathbb{Z}\left[t_{1}, \ldots, t_{n}\right]$ where $\left\{t_{1}, \ldots, t_{n}\right\}$ is a basis of the character group of $T_{n}$. Let $\iota_{v}: e_{v} \hookrightarrow \mathcal{F}_{n}$ denote the embedding. This induces $\iota_{v}^{*}: H_{T_{n}}^{*}\left(\mathcal{F}_{n}\right) \longrightarrow H_{T_{n}}^{*}\left(e_{v}\right)=\mathbb{Z}\left[t_{1}, \ldots, t_{n}\right]$. 
Theorem 4.1 Let $w \in W_{\infty}$. Take the smallest $n$ such that $w \in W_{n}$. Then for all $m \geq n$ and $v \in W_{m}$ we have

$$
\Phi_{v}\left(\mathfrak{C}_{w}\right)=\iota_{v}^{*}\left(\sigma_{w}^{T_{m}}\right) .
$$

Moreover we have $\Phi_{v}\left(\mathfrak{C}_{w}\right)=0$ if $v \notin W_{n}$.

This result says that the right hand side have a good stability property and the element $\mathfrak{C}_{w}$ represents the local datum simultaneously for all $v$.

Remark. Here we give just a very brief account of the proof of existence of $\left\{\mathfrak{C}_{w}\right\}_{w}$. At the heart of the construction there is a ring homomorphism

$$
\Phi=\left(\Phi_{v}\right)_{v}: R_{\infty} \longrightarrow \prod_{v \in W_{\infty}} \mathbb{Z}\left[t_{1}, t_{2}, \ldots\right]
$$

which is proved to be injective. We can describe the image $H_{\infty}$ of the map $\Phi$. The characterization conditions for the subring $H_{\infty}$ are a certain finiteness and a Goresky-Kottwitz-MacPherson type conditions. $H_{\infty}$ inherits a basis $\left\{\sigma_{w}^{(\infty)}\right\}$ parametrized by the elements in the infinite Weyl group $W_{\infty}$. The element $\sigma_{w}^{(\infty)}$ is a natural stable limit of $\xi$-functions of $\operatorname{Kostant}$ and $\operatorname{Kumar}(\mathrm{KK})$ for $H_{T_{n}}^{*}\left(\mathcal{F}_{n}\right)$. We have natural actions of left and right divided operators on $H_{\infty}$ which are compatible under the localization map $\Phi$. Then we can prove that the elements $\Phi^{-1}\left(\sigma_{w}^{(\infty)}\right) \in R_{\infty}$ solves the defining divided difference equations.

\subsection{Homomorphism $\pi_{n}$ and the classes $\beta_{i}$}

We introduce here a natural projection from the ring $R_{\infty}$ to equivariant cohomology of $\mathcal{F}_{n}$.

Proposition 4.2 The equivariant cohomology $H_{T_{n}}^{*}\left(\mathcal{F}_{n}\right)$ is canonically isomorphic to the ring

$$
R_{n}:=\mathbb{Z}\left[t_{1}, \ldots, t_{n}, z_{1}, \ldots, z_{n}\right] / I_{n},
$$

where $I_{n}$ is the ideal generated by the homogeneous parts of the relation

$$
\prod_{i=1}^{n}\left(1+z_{i}\right)\left(1-z_{i}\right)=\prod_{i=1}^{n}\left(1+t_{i}\right)\left(1-t_{i}\right) .
$$

Define the classes $\beta_{i} \in H_{T_{n}}^{2 i}\left(\mathcal{F}_{n}\right)$ by

$$
1+\beta_{1}+\beta_{2}+\cdots=\prod_{i=1}^{n} \frac{1-z_{i}}{1-t_{i}}=\prod_{i=1}^{n} \frac{1+t_{i}}{1+z_{i}}
$$

It is easy to see that the classes $\beta_{i}$ satisfy the same relations of $Q_{i}(x)$ 's. Thus we have the next proposition.

Proposition 4.3 There is a surjective ring homomorphism $\pi_{n}: R_{\infty} \longrightarrow H_{T_{n}}^{*}\left(\mathcal{F}_{n}\right)$ such that

$$
\pi_{n}\left(Q_{i}(x)\right)=\beta_{i}, \quad \pi_{n}\left(z_{i}\right)=\left\{\begin{array}{l}
z_{i} \quad \text { if } i \leq n \\
0 \quad \text { if } i>n
\end{array} \quad, \quad \pi_{n}\left(t_{i}\right)=\left\{\begin{array}{l}
t_{i} \quad \text { if } i \leq n \\
0 \quad \text { if } i>n
\end{array} .\right.\right.
$$


Remark. By the forgetful homomorphism $H_{T_{n}}^{*}\left(\mathcal{F}_{n}\right) \longrightarrow H^{*}\left(\mathcal{F}_{n}\right)$ the classes $\beta_{i}(1 \leq i \leq n)$ are mapped to the special Schubert classes in $H^{*}\left(L G_{n}\right) \subset H^{*}\left(\mathcal{F}_{n}\right)$ while $\beta_{i}(i>n)$ are mapped to zero. Note that $\beta_{i}(1 \leq i \leq n)$ in $H_{T_{n}}^{*}\left(\mathcal{F}_{n}\right)$ are not equal to the special (equivariant) Schubert classes.

By a direct calculation we have the following result.

Proposition 4.4 Let $v \in W_{n}$. The composition

$$
R_{\infty} \stackrel{\pi_{n}}{\longrightarrow} R_{n} \stackrel{\iota_{v}^{*}}{\longrightarrow} H_{T_{n}}^{*}\left(e_{v}\right)=\mathbb{Z}\left[t_{1}, \ldots, t_{n}\right] \hookrightarrow \mathbb{Z}\left[t_{1}, t_{2}, \ldots\right]
$$

is equal to $\Phi_{v}$.

Combined with this proposition and Theorem 4.1 we have the following.

Corollary 4.5 We have

$$
\pi_{n}\left(\mathfrak{C}_{w}\right)=\left\{\begin{array}{l}
\sigma_{w}^{T_{n}} \quad \text { if } w \in W_{n} \\
0 \quad \text { otherwise }
\end{array} .\right.
$$

\section{Appendix}

For reader's convenience we present some basic results on Ivanov's $Q$-functions. We use slightly different notation from that of (Iv). Let $t=\left(t_{1}, t_{2}, \ldots\right)$ be indeterminates. Note that the parameters $a_{i}$ used in (Iv) are related to our convention by $a_{i+1}=t_{i}$ with $a_{1}=0$. Consider the ring $\mathbb{Z}[t] \otimes_{\mathbb{Z}} \Gamma$ where $\mathbb{Z}[t]=\mathbb{Z}\left[t_{1}, t_{2}, \ldots\right]$. Let $\lambda=\left(\lambda_{1}>\cdots>\lambda_{r}>0\right)$ be a strict partition. Define

$$
t_{\lambda}=\left(t_{\lambda_{1}}, \ldots, t_{\lambda_{r}}, 0,0, \ldots\right) .
$$

Let $v \in W_{\infty}$ be the maximal Grassmannian element corresponding to $\lambda \in \mathcal{S P}$. Define

$$
H_{\lambda}(t)=\prod_{(i, j) \in \lambda}(t \overline{v(i)}+t \overline{v(j)}) .
$$

Proposition $5.1((I v))$ For any strict partition $\lambda$, the factorial $Q$-function $Q_{\lambda}(x \mid t)$ is a unique element of $\mathbb{Z}[t] \otimes_{\mathbb{Z}} \Gamma$ that satisfies following conditions:

1. $Q_{\lambda}(x \mid t)$ is homogeneous of degree $|\lambda|=\sum_{i=1}^{r} \lambda_{i}$,

2. $Q_{\lambda}(x \mid t)=Q_{\lambda}(x)+$ lower order terms in $x$,

3. $Q_{\lambda}\left(t_{\mu} \mid t\right)=0$ unless $\lambda \subset \mu$,

4. $Q_{\lambda}\left(t_{\lambda} \mid t\right)=H_{\lambda}(t)$.

Remark. In (IN) a combinatorial expression in terms of 'excited Young diagrams' for $Q_{\lambda}\left(t_{\mu} \mid t\right)$ was obtained.

Proposition $5.2((I v))$ The factorial $Q$-functions $Q_{\lambda}(x \mid t)(\lambda \in \mathcal{S P})$ form a $\mathbb{Z}[t]$-basis of $\mathbb{Z}[t] \otimes_{\mathbb{Z}} \Gamma$. 
Proposition $5.3((I v))$ For any strict partition $\lambda=\left(\lambda_{1}>\cdots>\lambda_{r} \geq 0\right)$ with $r$ even, we have

$$
Q_{\lambda}(x \mid t)=\operatorname{Pf}\left(Q_{\lambda_{i}, \lambda_{j}}(x \mid t)\right)_{1 \leq i<j \leq r} .
$$

Note. After the present work was completed we were informed that A. Kirillov (K) had introduced double Schubert polynomials of type $B$ (and $C$ ) in 1994 by using Yang-Baxter operators (cf. (FK)), independently to us, although the geometric interpretation as in the present article had not been known. The approach is quite different from ours, nevertheless the polynomials seem to be essentially the same as ours; after a suitable identification of variables (see remark in 3.1).

\section{Acknowledgements}

We are grateful to $\mathrm{H}$. Tamvakis and S. Billey for enlightening conversations that motivated the present work. We also would like to thank A. Kirillov for explaining us his previous unpublished works.

\section{References}

[A] A. Arabia, Comomologie $T$-équivariante de $G / B$ pour an groupe $G$ de Kac-Moody, C. R. Acad. Sci. Paris Sér. I. Math. 302 (1986) 631-634.

[BGG] I. N. Bernstein, I. M. Gelfand, and S. I. Gelfand, Schubert cells and cohomology of the spaces $G / P$, Russ. Math. Surv. 28 (1973), no. 3, 1-26.

[B1] S. Billey, Transition equations for isotropic flag manifolds, Discrete Math. 193 (1998) 69-84.

[B2] S. Billey, Kostant polynomials and the cohomology ring for $G / B$, Duke Math. J. 96, no. 1 (1999), 205-224.

[BH] S. Billey and M. Haiman, Schubert polynomials for the classical groups, J. Amer. Math. Soc. 8 (1995), no. 2, 443-482.

[D] M. Demazure, Désingularization des variétés de Schubert generalisées, Ann. Sci. École Norm. Sup. 7 (1974), 53-88.

[FR] L. Fehér and R. Rimányi, Schur and Schubert polynomials as Thom polynomials - cohomology of moduli spaces, Cent. Eur. J. Math. 1 (2003), no. 4, 418-434 (electronic).

[FK] S. Fomin and A. N. Kirillov, Combinatorial $B_{n}$-analogues of Schubert polynomials, Trans. Amer. Math. Soc. 348 (1996), no. 9, 3591-3620.

[F1] W. Fulton, Flags, Schubert polynomials, degeneracy loci, and determinantal formulas, Duke Math. J. 65 (1991) 381-420.

[F2] W. Fulton, Determinantal formulas for orthogonal and symplectic degeneracy loci, J. Diff. Geom., 43 (1996), 276-290. 
[GKM] M. Goresky, R. Kottwitz, and R. MacPherson, Equivariant cohomology, Koszul duality, and the localization theorem, Invent. Math. 131 (1998) 25-83.

[I] T. Ikeda, Schubert classes in the equivariant cohomology of the Lagrangian Grassmannian, Adv. Math. 215 (2007), 1-23.

[IMN] T. Ikeda, L.Mihalcea and H. Naruse, Double Schubert polynomials for the classical groups, in preparation.

[IN] T. Ikeda and H. Naruse, Excited Young diagrams and equivariant Schubert calculus, preprint. arXiv: math/0703637

[Iv] V. N. Ivanov, Interpolation analogue of Schur $Q$-functions, Zap. Nauc. Sem. S.-Peterburg. Otdel. Mat. Inst. Steklov. 307 (2004) 99-119.

[Ka] M. Kazarian, On Lagrange and symmetric degeneracy loci, preprint. http://www.newton.cam.ac.uk/preprints2000.html

[K] A. Kirillov, private communication.

[KK] B. Kostant and S. Kumar, The nil Hecke ring and cohomology of $G / P$ for a Kac-Moody group $G$, Adv. Math. 62 (1986) 187-237.

[KT] A. Kresch and H. Tamvakis, Double Schubert polynomials and degeneracy loci for the classical groups, Ann. Inst. Fourier (Grenoble) 52 (2002), no. 6, 1681-1727.

[L] A. Lascoux, Classes de Chern des variétés de drapeaux, C. R. Acad. Sci. Paris. Sér. I Math. 295 (1982), no. 1, 1-43.

[LS] A. Lascoux and M.-P. Schützenberger, Polynômes de Schubert, C. R. Acad. Sci. Paris Sér. I Math. 294 (1982), no. 13, 447-450.

[M] I. G. Macdonald, Symmetric functions and Hall polynomials, 2nd edition, Oxford Univ. Press, Oxford 1995.

[Mi] L. C. Mihalcea, Giambelli formulae for the equivariant quantum cohomology of the Grassmannian, Trans. Amer. Math. Soc. 360 (2008), no. 5, 2285-2301.

[P] P. Pragacz, Algebro-geometric applications of Schur $S$ - and $Q$-polynomials, in Séminaire d'Algèbre Dubreil-Malliavin 1989-1990, Springer Lecture Notes in Math. 1478 (1991) 130-191. 